Service social

\title{
Un contexte qui a changé
}

\section{André Beaudoin, Richard Lefrançois et Francine Ouellet}

Volume 35, numéro 1-2, 1986

Recherche - Action - Évaluation

URI : https://id.erudit.org/iderudit/706291ar

DOI : https://doi.org/10.7202/706291ar

Aller au sommaire du numéro

Éditeur(s)

École de service social de l'Université Laval

ISSN

1708-1734 (numérique)

Découvrir la revue

Citer ce document

Beaudoin, A., Lefrançois, R. \& Ouellet, F. (1986). Un contexte qui a changé.

Service social, 35(1-2), 12-14. https://doi.org/10.7202/706291ar d'utilisation que vous pouvez consulter en ligne.

https://apropos.erudit.org/fr/usagers/politique-dutilisation/ 


\section{AVANT-PROPOS}

\section{Un contexte qui a changé}

La conjoncture historique et le contexte socio-politique des années 1960, qui ont préparé au Québec l'avènement de l'ÉtatProvidence, ont accentué la nécessité de la recherche. Grâce en particulier à l'expansion du système de distribution de services sociaux et sanitaires, au déploiement des professions orientées vers l'intervention sociale, et sous l'impulsion des mouvements sociaux, la recherche sociale s'est progressivement taillée une place.

Or, depuis la situation économique défavorable des dernières années, la politique sociale de l'État, qui tend à se réarticuler dans une optique de désinstitutionnalisation, cherche à privilégier une philosophie nouvelle axée sur la prévention et le recours à des stratégies alternatives aux solutions professionnelles et institutionnelles de prise en charge des clientèles cibles.

Un tel retournement s'accompagne en même temps d'une sensibilisation accrue aux problèmes de gestion des programmes maintenus et d'un plus grand souci de contrôle des activités relevant de l'intervention sociale.

Jeune de tradition, la recherche évaluative au Québec se rattache encore à ces préoccupations premières. La création du Conseil québécois de la recherche sociale en 1980 et, un peu plus tard, celle de la Direction de l'évaluation des programmes au Ministère des affaires sociales (mars 1982), confirment cette nécessité de questionnement, de contrôle et d'évaluation vue comme processus informatif eu égard à une décision éventuelle.

Comparativement à nos voisins américains qui parlent et font de l'évaluation depuis plus de deux décennies, les Québécois s'en préoccupent surtout depuis le début des années 1980 et leurs productions sont relativement restreintes.

Nous assistons donc présentement à une remontée de la recherche évaluative, à un désir de production de recherche et à un souci de raffinement méthodologique. Ce courant, toutefois, ne peut être étranger aux transformations fondamentales qui s'opèrent dans les 
politiques sociales de l'État et, conséquemment, au sein des pratiques sociales. En même temps que le réseau formel de distribution des services sociaux vit à l'ère des restrictions, de nouvelles pratiques dites "alternatives" s'installent. À des fins de contrôle, de rationalisation, le système presse les chercheurs d'évaluer les programmes, les services, les interventions; d'autre part, de l'intérieur, les intervenants questionnent leurs pratiques.

On assiste donc à une transformation notoire dans le discours et dans les pratiques de recherche. Certaines recherches évaluatives sont commandées de l'extérieur tandis que d'autres naissent de l'intérieur. Certaines opèrent à partir du paradigme expérimental traditionnellement reconnu, d'autres se fondent sur une approche plus naturaliste et qualitative. Bien que ces perspectives semblent se confronter, elles ont néanmoins toutes leur place dans la conjoncture actuelle; le développement d'une recherche doit passer par la confrontation scientifique qui enrichit le discours et la pratique.

Il est par ailleurs intéressant de noter que cette préoccupation en matière d'évaluation déborde le contexte québécois. Par exemple, le dernier numéro de la revue française POUR porte sur le thème de "L'évaluation au pouvoir". Il y a donc lieu de conclure à la pertinence sociale du discours sur l'évaluation, lequel dépasse largement les frontières du Québec et du Canada.

Le présent numéro veut donc refléter ce changement de rôle de la recherche sociale, et plus particulièrement de la recherche évaluative. Celle-ci répond-elle maintenant à deux niveaux différents d'objectifs ? A-t-elle tendance à se rapprocher de la recherche-action? Assistonsnous à la redéfinition de la recherche évaluative? Voilà certaines questions auxquelles le lecteur cherchera une réponse au travers de ces pages.

Ce débat sera tout d'abord abordé sous l'angle de ses fondements épistémologiques. Ricardo Zúñiga touche la question du rôle des évaluateurs, des chercheurs et des travailleurs sociaux. Régis Blais soulève ensuite l'épineuse question des résistances liées au processus de l'évaluation et concrétise son questionnement dans le contexte de l'évaluation des programmes dans les C.L.S.C.

Un second bloc d'articles se veut davantage méthodologique. Certains modèles particuliers à l'évaluation sont donc abordés, tels que celui de l'évaluation sur cas unique présenté par André Beaudoin et celui de l'évaluation institutionnelle que décrit Jacques Plante. Nous n'avons toutefois pas la prétention de vouloir faire de cette section "la" méthodologie en recherche évaluative. D’autres modèles existent; ceux-ci ne sont que des exemples d'un univers plus large. 
Une attention spéciale est ensuite accordée à des projets de recherche qui témoignent d'expériences concrètes. C'est le cas, notamment, des articles de Francine Ouellet, et de Marie Bruneau, Michel Laflamme et Marie Josée Linteau, qui s'inscrivent dans le courant des évaluations de programme. Quant à Lise Darveau-Fournier et Alice Home, ainsi qu'à Maryse Rinfret-Raynor, Michèle Larouche et Ann Pâquet-Deehy, elles rejoignent davantage le courant des évaluations d'interventions ou de stratégies d'intervention.

Enfin, deux autres articles se présentent comme des bilans. Celui de Laurent Chambaud, Robert Mayer et Gabrielle Richard est un bilan critique des recherches-actions québécoises; ce regard furtif vers ce type de recherche nous commande de repenser l'alliance actionévaluation ou praticien-chercheur. Un dernier article, d'André Beaudoin, Richard Lefrançois et Francine Ouellet, résume les enjeux actuels de la recherche évaluative, en définit les balises en termes d'applications et propose quelques pistes de réflexion qui en favoriseraient une conception renouvelée.

André BEAUDOIN

Richard LEFRANÇOIS

Francine OUeLlet 